\title{
Error-Rate Dependence of Non-Bandlimited Signals with Finite Rate of Innovation
}

\author{
Ivana Jovanović and Baltasar Beferull-Lozano \\ Audio-Visual Communications Laboratory, EPFL, CH-1015 Lausanne, Switzerland \\ $\{$ Ivana.Jovanovic, Baltasar.Beferull\}@epfl.ch
}

\begin{abstract}
Recent results in sampling theory [1] showed that perfect reconstruction of non-bandlimited signals with finite rate of innovation can be achieved performing uniform sampling at or above the rate of innovation. We study analog-to-digital (A/D) conversion of these signals, introducing two types of ovrsampling and consistent reconstruction.
\end{abstract}

In this work, we consider periodic streams of $K$ Diracs, that is, $x(t)=\sum_{k \in \mathcal{Z}} c_{k} \delta\left(t-t_{k}\right)=\sum_{m \in \mathcal{Z}} X[m] e^{j(2 \pi m t) / \tau}$ with $X[m]=\frac{1}{\tau} \sum_{k=0}^{K-1} c_{k} e^{-j\left(2 \pi m t_{k}\right) / \tau}$ and period $\tau$, where $t_{k+K}=$ $t_{k}+\tau, c_{k+K}=c_{k}, \forall k \in \mathcal{Z}$ and $\delta(t)$ denotes a Dirac delta function. The signal has $2 K / \tau$ degrees of freedom per unit of time. Time positions $\left\{t_{k}\right\}_{k=0}^{K-1}$ and weights $\left\{c_{k}\right\}_{k=0}^{K-1}$ can be perfectly reconstructed by first applying a sinc sampling kernel $h_{B}(t)=B \operatorname{sinc}(B t)$ with bandwidth $[-B \pi, B \pi]$, thus obtaining $y(t)=x(t) * h_{B}(t)$, and then taking the $N \geq 2 M+$ 1 uniform samples $y_{n}=\sum_{m=-M}^{M} X[m] e^{j 2 \pi m n T / \tau}$ with $T=$ $\tau / N$, such that $B \tau=2 M+1 \geq 2 K+1$. After computing $2 K+1$ Fourier coefficients from $y_{n}$, we apply annihilating filter method. From the roots of annihilating filter $\left\{u_{k}=\right.$ $\left.e^{-j 2 \pi t_{k} / \tau}\right\}_{k=0}^{K-1}$ we get the $K$ time positions $\left\{t_{k}\right\}_{k=0}^{K-1}$, while the weights $\left\{c_{k}\right\}_{k=0}^{K-1}$ can then be directly computed.

We overcome the error in amplitude of the samples $\left\{y_{n}\right\}_{k=0}^{K-1}$, introduced due to the quantization, by performing two types of oversampling. The first one, oversampling in time, consists of taking more samples of $y(t)$ than necessary, so that $N>2 M+1$, with oversampling ratio $R_{t}=N / 2 M+1$. In the second one, oversampling in frequency, we extend the bandwidth $B=2 M+1$ so that it is greater than the rate of innovation, that is, $M>K$, with oversampling ratio $R_{f}=(2 M+1) /(2 K+1)$.

We also introduce the concept of consistent reconstruction for these types of signals. The idea is to exploit all the $a$ priori knowledge of the original signal and the quantization process itself. We first define the three sets of constraints on which we have to project. Set $\boldsymbol{S}_{\mathbf{1}}$ is defined by the quantization operation and consists of the quantization bins in which the samples $\left\{y_{n}\right\}_{n=0}^{N-1}$ lie. Set $\boldsymbol{S}_{\mathbf{2}}$ is the set of continuoustime periodic signals bandlimited to $[-B \pi, B \pi]$ to which $y(t)$ belongs.

Based on this, satisfying these two sets we provide a first level of accuracy, weak consistency, which we achieve by iterating projections $\boldsymbol{P}_{\mathbf{1}}$ and $\boldsymbol{P}_{\mathbf{2}}$.

Def. 1 A reconstruction $\hat{x}(t)$ satisfies weak consistency $(W C)$ iff it is obtained from a signal $\hat{y}(t)$ such that: a) the samples $\left\{\hat{y}_{n}\right\}_{n=0}^{N-1}$ lie in the same quantization bins as the original ones, $\left.\left\{\hat{y}_{n}\right\}_{n=0}^{N-1} \in \boldsymbol{S}_{\mathbf{2}}, b\right) \hat{y}(t) \in \boldsymbol{S}_{\mathbf{1}}$.

Proj. $\boldsymbol{P}_{\mathbf{1}}$ : For every estimate $\hat{y}_{n}^{i}, \hat{y}_{n}^{i+1}=\boldsymbol{P}_{\mathbf{1}}\left(\hat{y}_{n}^{i}\right)$ is given by: a) $\hat{y}_{n}^{i+1}=\hat{y}_{n}^{i}$ if $\left.\hat{y}_{n}^{i} \in \boldsymbol{S}_{\mathbf{1}}, 2\right)$ else, $\hat{y}_{n}^{i+1}$ is set to the bound of

\footnotetext{
${ }^{1}$ This work was supported (in part) by the National Competence Center in Research on Mobile Information and Communications Systems (NCCR-MICS), a center supported by Grant 5005-67322.
}

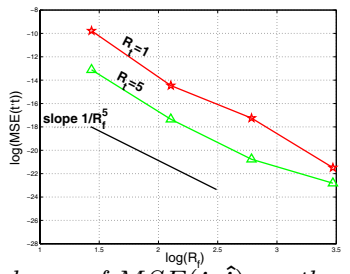

Figure 1: Dependence of $M S E(\boldsymbol{t}, \hat{\boldsymbol{t}})$ on the factors $R_{t}$ and $R_{f}$. the quantization interval in $\boldsymbol{S}_{\mathbf{1}}$ closest to $\hat{y}_{n}^{i}$.

Proj. $\boldsymbol{P}_{\mathbf{2}}$ : Given an estimate $\hat{y}^{i}(t)$, the new estimate $\hat{y}^{i+1}(t)=$ $\boldsymbol{P}_{\mathbf{2}}\left(\hat{y}^{i}(t)\right)$ is obtained by low-pass filtering $\hat{y}^{i}(t)$, that is $\hat{y}^{i+1}(t)=\hat{y}^{i}(t) * h_{B}(t)$. The particular structure of the signal $x(t)$ defines the third set which, together with previous two sets, is used to enforce a stronger sense of consistency. The Set $\boldsymbol{S}_{\mathbf{3}}$ is the set of Fourier coefficients that originate from a periodic stream of Diracs, $X[m]=\frac{1}{\tau} \sum_{k=0}^{K-1} c_{k} e^{-j 2 \pi m t_{k} / \tau}$.

Def. 2 A reconstruction $\hat{x}(t)$ satisfies strong consistency (SC) iff: a) it satisfies weak consistency, b) $\hat{y}_{n}=\left.h_{b}(t) * \hat{x}(t)\right|_{n T}$ where $\hat{x}(t)$ is a periodic stream of $K$ Diracs.

Proj.P. $P_{\mathbf{3}}$ : Given a set of estimated Fourier coefficients $\hat{\boldsymbol{X}}^{i}$, the projection $\boldsymbol{P}_{\mathbf{3}}$ provides $\left\{\left(\hat{t}_{k}^{i+1}, \hat{c}_{k}^{i+1}\right)\right\}_{k=0}^{K-1}$ and a set of Fourier coefficients $\hat{\boldsymbol{X}}^{i+1}$ such that $\hat{X}^{i+1}[m]=$ $\frac{1}{\tau} \sum_{k=0}^{K-1} \hat{c}_{k}^{i+1} e^{-j 2 \pi m \hat{t}_{k}^{i+1} / \tau}$.

Theorem 1 Given $x(t)$, for any reconstruction $\hat{x}(t)$ obtained using $\boldsymbol{P}_{\mathbf{3}}$ and which satisfies $W C$, there exist $\xi \geq 1$ such that if $R_{t}, R_{f} \geq \xi$, there is a constant $c>0$ which depends only on $x(t)$ and not on $R_{t}$ and $R_{f}$, and $M S E(t, \hat{\boldsymbol{t}}) \leq \frac{c}{R^{3} R^{2}}$. (see [3])

For the method that achieve $S C$ the experinfertital results show, a performance of $M S E(\boldsymbol{t}, \hat{\boldsymbol{t}})=O\left(1 / R_{t}^{2} R_{f}^{5}\right)$ for time positions (Fig. 1), with parameters: $K=2, \tau=10, t_{k} \in$ $(0, \tau], c_{k} \in[-1,1]$.

We also compare two types of encoding, the traditional one, pulse-code modulation encoding $(\mathrm{PCM})$ and the alternative one, based on threshold crossing encoding (TC) [2], and investigate in the dependence of the bit rate on the oversampling factors $R_{t}$ and $R_{f}$, and the quantization step size $\Delta$. The following table, shows the theoretical results for the bit rate and also both theoretical and experimental results for the MSE of time positions.

\begin{tabular}{|c||c||c||c||}
\hline & Bit rate $(\mathrm{b})$ & $M S E-W C$ & $M S E-S C$ \\
\hline \multirow{3}{*}{$\mathrm{TC}$} & $O\left(\log _{2} R_{t}\right)$ & $O\left(1 / R_{t}^{2}\right)$ & $O\left(1 / R_{t}^{2}\right)$ \\
& $O\left(R_{f} \log _{2} R_{f}\right)$ & $O\left(1 / R_{f}^{3}\right)$ & $O\left(1 / R_{f}^{5}\right)$ \\
& $O(1 / \Delta)$ & $O\left(\Delta^{2}\right)$ & $O\left(\Delta^{2}\right)$ \\
\hline \multirow{3}{*}{$\mathrm{PCM}$} & $O\left(R_{t}\right)$ & $O\left(1 / R_{t}^{2}\right)$ & $O\left(1 / R_{t}^{2}\right)$ \\
& $O\left(R_{f} \log _{2} R_{f}\right)$ & $O\left(1 / R_{f}^{3}\right)$ & $O\left(1 / R_{f}^{5}\right)$ \\
& $O\left(\log _{2}(1 / \Delta)\right)$ & $O\left(\Delta^{2}\right)$ & $O\left(\Delta^{2}\right)$ \\
\hline
\end{tabular}

Notice that oversampling in time provide the error-rate dependence $\left(O\left(2^{-2 \alpha b}\right)\right)$ that can be obtain by decreasing the step size $\left(O\left(2^{-2 \beta b}\right)\right)$.

REFERENCES
[1] M. Vetterli, P. Marziliano, and T. Blu "Sampling signals with finite rate of innovation" IEEE Trans. on Signal Proc., vol. 50, no. 6,

[2] Pp. T. Thao and M. Vetterli, "Deterministic Analysis of Oversampled A/D Conversion and Decoding Improvement Based on Consistent Estimates" IEEE Trans. on Signal Proc., vol. 42, no. 3, pp. $519-531,1994$.

[3] I. Jovanović and B. Beferull-Lozano, "Oversampled $A / D$ Conversion of Non-Bandlimited Signals with Finite Rate of Innovation", To be submitted to IEEE Trans. on Signal Proc. 